\title{
High resolution optical time-domain reflectometry based on correlation utilizing an all-fiber chaotic source
}

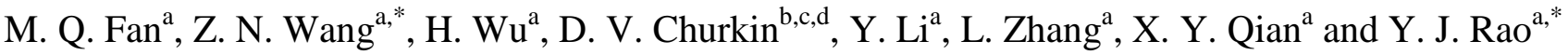 \\ ${ }^{a}$ Key Lab of Optical Fiber Sensing \& Communications, University of Electronic Science \\ \&Technology of China, Chengdu, Sichuan, China 611731 \\ ${ }^{\mathrm{b}}$ Institute of Automation and Electrometry SB RAS, Novosibirsk 630090, Russia \\ ${ }^{c}$ Novosibirsk State University, Novosibirsk 630090, Russia \\ ${ }^{\mathrm{d}}$ Aston Institute of Photonic Technologies, Aston University, Birmingham, B4 7ET, UK
}

\begin{abstract}
We propose a high-resolution optical time domain reflectometry (OTDR) based on an all-fiber supercontinuum source. The source simply consists of a laser with moderate power and a section of fiber which has a zero dispersion wavelength near the laser's central wavelength. Spectrum and time domain properties of the source are investigated, showing that the source has great capability in nonlinear optics, such as correlation OTDR. We analyze one of the key factors limiting the operational range of such an OTDR, i.e., sampling time. Finally, we experimentally demonstrate a correlation OTDR with $25 \mathrm{~km}$ sensing range and $5.3 \mathrm{~cm}$ spatial resolution, as a verification of theoretical analysis.
\end{abstract}

Keywords: Fiber measurements, Optical time domain reflectometry, Rayleigh scattering.

\section{INTRODUCTION}

Chaotic sources have attracted much attention due to its unique characteristics and the huge potential for various applications including physical random bit generation [1], secure communication [2], chaotic lidar [3] and so on. As an example of the applications of electrical driven chaotic source, in 2007, a cross-correlation OTDR [4] was proposed utilizing a pseudo-random pulse sequence as the signal. However, its measurement accuracy is still limited by the bottleneck of the pseudo-random modulation bandwidth, and it is difficult and costly to generate broadband electrical random codes. For the above reasons, electronic chaotic sources are hard to compete with optical chaotic sources which have advantages of wider bandwidth and faster chaotic dynamics.

Followed this work, Y. Wang et al. demonstrated a $6 \mathrm{~cm}$ spatial correlation OTDR based on a multi-GHz optical chaotic source utilizing LD laser [5]. This is the first proof-of-concept experiment of correlation OTDR by utilizing the optical chaotic source. However, that work only presented a $140 \mathrm{~m}$ detection ranging. There is always tradeoff between the spatial resolution and detection range.

In this work, we propose a high-resolution correlation OTDR based on an all fiber supercontinuum (SC) source. The fluctuation and auto-correlation properties of such SC sources are investigated for the first time. It is demonstrated that the SC source is a powerful tool to generate ultra-wideband fluctuation in time domain, which is an ideal source for correlation OTDR, and its potential to achieve millimeter-scale resolution is shown. On the other hand, one of the limiting factors for realizing long-range correlation OTDR is analyzed, i.e. sampling time. We analyze the potential methods to overcome the bottleneck of operation range. As a result, we experimentally demonstrate a correlation OTDR which has 25 kilometers fiber fault location range with $5.3 \mathrm{~cm}$ spatial resolution, with only 400 us data acquisition time.

\section{CHARACTERISTICS OF THE SUPERCONTINUUM SOURCE}

The schematic setup of the proposed system is shown in Fig. 1. The proposed correlation OTDR system mainly consists of two parts (i.e. SC and OTDR). As the box diagram in Fig. 1 shows, the SC simply consists of a $1455 \mathrm{~nm}$ quasi-CW Raman fiber laser and $16 \mathrm{~km}$ TrueWave (TW) fiber.

Fifth Asia-Pacific Optical Sensors Conference, edited by Byoungho Lee, Sang-Bae Lee,

Yunjiang Rao, Proc. of SPIE Vol. 9655, 96553J - (c) 2015 SPIE

CCC code: 0277-786X/15/\$18 - doi: 10.1117/12.2184238 


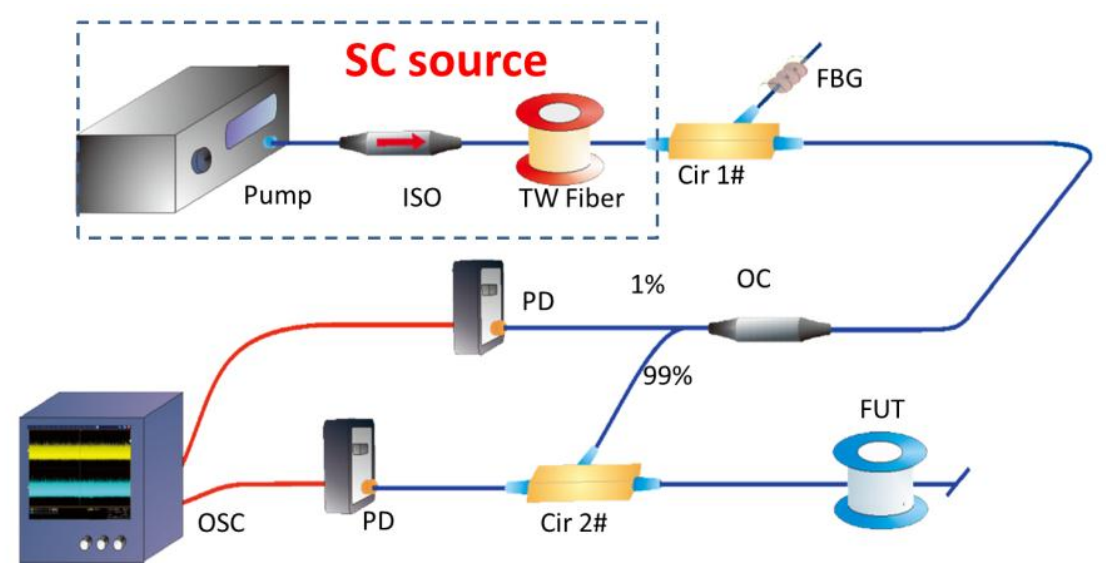

Fig. 1. Schematic setup (ISO: isolator; TW Fiber: TrueWave Fiber; SC source: supercontinuum source;

Cir: circulator; FBG: fiber Bragg grating; OC: optical coupler; PD: photo-detector; FUT: fiber under test; OSC: oscilloscope).

The SC spectra recorded at the end of the TW fiber are shown in Fig. 2. It should be noted that, when pump power reaches to $0.95 \mathrm{~W}$, spectral components extended to $1550 \mathrm{~nm}$ region are generated. If the pump power is increased to $1.48 \mathrm{~W}$, the generated $\mathrm{SC}$ has the widest bandwidth, i.e., $141 \mathrm{~nm}$ within $10 \mathrm{~dB}$ range. By further increasing pump power, the bandwidth of generated SC would be reduced. The reason is that more powerful pump stimulates higher-order Stokes waves and enhances total pump-Stokes conversion efficiency, thus shorter wavelength photons are significantly depleted.

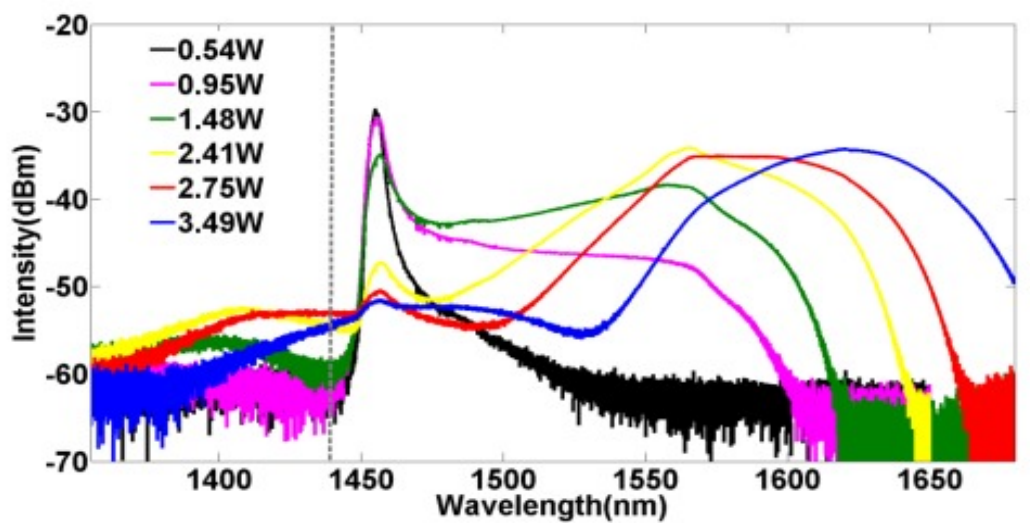

Fig. 2. Spectrum evolution with pump power (the vertical dotted line corresponds to the zero-dispersion wavelength of the TW fiber).
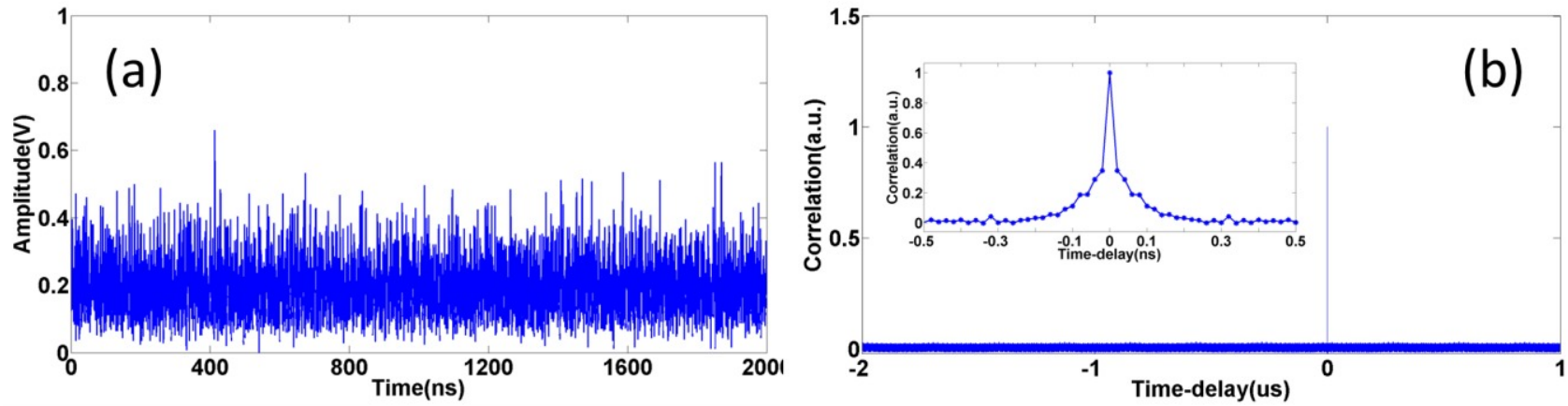

Fig. 3. (a) Time series of generated SC (with 1.48W pump power); (b) ACF of SC, insert: magnified peak of ACF.

We investigate the source in time domain (filtered with $0.26 \mathrm{~nm}$ FBG). A $45 \mathrm{GHz} \mathrm{PD}$ and an oscilloscope with $25 \mathrm{GHz}$ bandwidth and 50Gs/s sampling rate are used. The time series are shown in Fig. 3(a). It shows that the SC has time dynamics with high-contrast fluctuations which is a favorable condition for correlation analysis. We calculate the autocorrelation function (ACF) with DC removed, as shown in Fig. 3(b). Narrow-width ACF peak ( 20ps, corresponding to 
$\sim 2 \mathrm{~mm}$ spatial resolution for OTDR) could result in the ultra-fine resolution for correlation OTDR, up to the limit of the bandwidth of the PD and the oscilloscope (ignoring the signal distortion in fiber). Overall, the demonstrated SC source could be an important member in the family of chaotic sources because of its stochastic temporal properties.

\section{DISCUSSIONS FOR PERFORMANCE ENHANCEMENT OF CORRELATION OTDR}

With the increase of sensing range, optical signal to noise ratio (OSNR) of the detected signal would decrease thus it will be difficult to recognize the peaks among correlation trace. To extend the detection range of correlation OTDR, the signal quality must be improved.

Increasing sampling time is a direct way to enhance system performance. In correlation OTDR, the crosscorrelation procedure is essentially matched filtering. Assuming $\mathrm{x}(\mathrm{t})$ is the signal, the maximum achievable SNR can be expressed as [6]

$$
S N R=\frac{\int_{-\infty}^{+\infty}|x(t)|^{2} d t}{N_{0}}
$$

where $\mathrm{N}_{0}$ is power spectral density of white noise. On the other hand, the energy of signal $\mathrm{x}(\mathrm{t})$ is as $E=\int_{-\infty}^{+\infty}|x(t)|^{2} d t$. Finally, we could obtain that the maximum achievable $\mathrm{SNR}=\mathrm{E} / \mathrm{N}_{0}$. It depends only on the energy of the waveform. Thus, if the sampling time is increased, SNR of correlation OTDR would be increased linearly. As the verification, we demonstrate a $1 \mathrm{~km}$ sensing range experiment without any amplification. Fig. 4 shows SNR varies with different sampling times. Basically, the experiment result is qualitatively in accordance with the theoretical analysis.

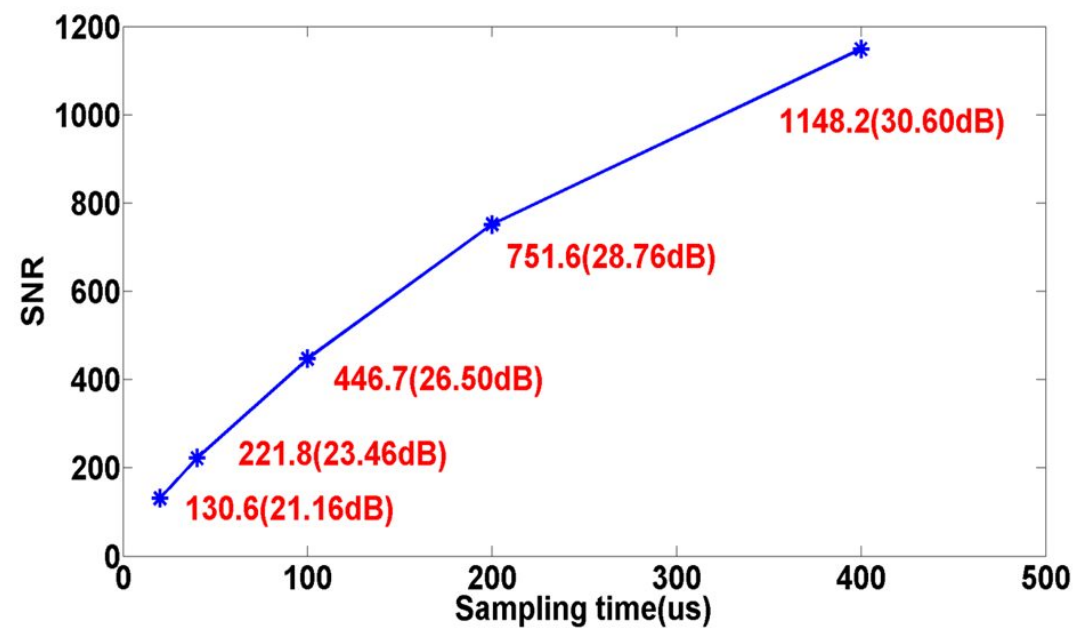

Fig. 4 Calculated SNR versus sampling time

\section{EXPERIMENTAL DEMONSTRATION WITH CORRELATION OTDR}

Finally, we establish an experimental system following Fig. 1. The combination of 1550nm fiber Bragg grating (FBG) and a circulator (CIR1) is act as a narrow bandwidth filter. The $3 \mathrm{~dB}$ bandwidth of the filter is $0.6 \mathrm{~nm}$. After port3 of the CIR1, a 1:99 optical coupler is used to split the filtered light into two branches. One branch (1\%) is used as the reference light detected by a $1 \mathrm{GHz}$ photo-detector, while the other branch $(99 \%)$ transmission light acts as the chaotic probe light of OTDR. The fiber under test is about $25 \mathrm{~km}$ standard single mode fiber (SMF) with $0.18 \mathrm{~dB} / \mathrm{km}$ loss at $1550 \mathrm{~nm}$. The reflected signal is detected by a $1 \mathrm{GHz}$ photo-detector which is similar to the detector placed in $1 \%$ branch. A FC/APC connector connects to the fiber end port thus the connector is used to emulate fiber-fault.

The pump power of SC is $31.6 \mathrm{dBm}$, the power of $1 \%$ branch of the filtered chaotic light is $-17 \mathrm{dBm}$, while the $99 \%$ branch power is $0.82 \mathrm{dBm}$. The total reflected light power detected by the detector is $-25 \mathrm{dBm}$. The signals of the two detectors are simultaneously recorded for $400 \mathrm{us}$ by a multi-channel oscilloscope with $25 \mathrm{GHz}$ sampling rate. The recorded data is processed with cross correlation algorithm and the fiber fault could be located by the correlation trace. Fig. 5 shows the normalized correlation OTDR trace. The position of the peak $(25447.541 \mathrm{~m})$ is corresponding to the open end of the FC/PC connector. The inset of fig. 5 shows the magnified correlation peaks. From the magnified trace, we also identify the spatial resolution of the system as $5.3 \mathrm{~cm}$. 


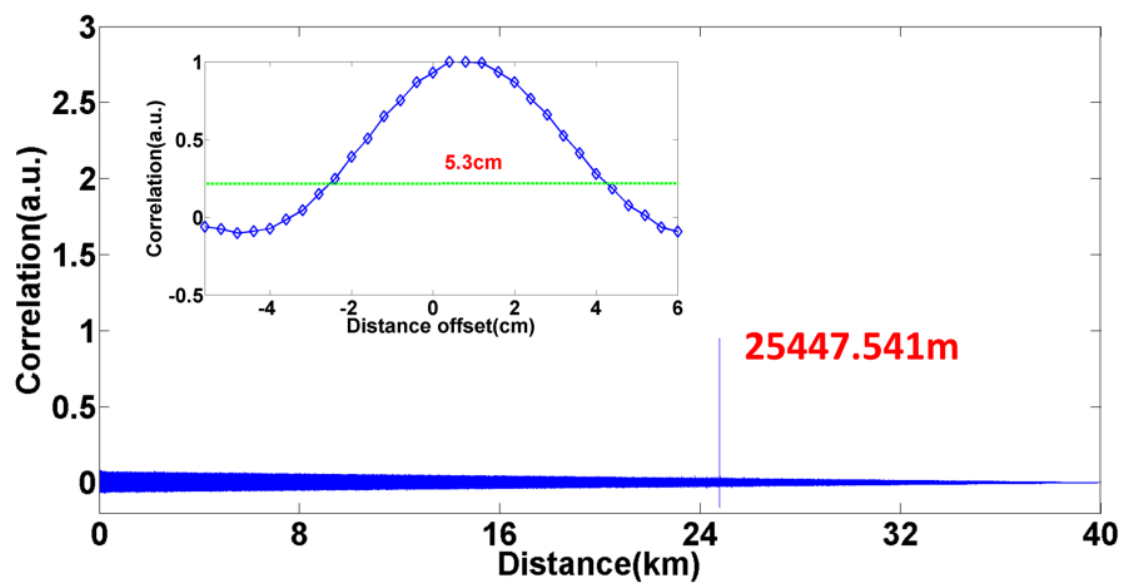

Fig. 5 Experimental result of fault at $25447.541 \mathrm{~m}$; inset: magnified peaks of the correlation trace.

\section{CONCLUSION}

In summary, a high-resolution correlation OTDR based on all-fiber SC source is proposed. The temporal properties of $\mathrm{SC}$ are investigated, showing that the SC is a promising source of chaotic radiation for applications such as correlation OTDR. The sampling time as the key factor limiting the sensing range of correlation OTDR is analyzed. The result shows that increasing sampling time is an effective way to enhance the SNR of correlation trace. Finally, we realize a centimeter level spatial resolution and $25 \mathrm{~km}$ sensing range OTDR measurement, which is a significant improvement compare to the conventional OTDR.

\section{ACKNOWLEDGMENTS}

This work is supported by Natural Science Foundation of China (61205048, 61290312), RFDP (20120185120003), and PCSIRT (IRT1218), and the 111 project (B14039). The authors also thank Prof. Xingwen Yi in UESTC and Chengdu Best Xingbang Technology Limited for providing the oscilloscopes.

\section{REFERENCES}

[1] A. Uchida, K. Amano, M. Inoue, K. Hirano, S. Naito, H. Someya, I. Oowada, T. Kurashige, M. Shiki, S.Yoshimori, K. Yoshimura, and P. Davis, "Fast physical random bit generation with chaotic semiconductorlasers," Nat. Photonics 2, 728-732 (2008).

[2] A. Argyris, D. Syvridis, L. Larger, V. Annovazzi-Lodi, P.Colet, I. Fischer, J. Garcia-Ojalvo, C. R. Mirasso, L. Pesquera, and K. A. Shore, "Chaos-based communications at high bit rates using commercial fibre-optic links", Nature 438, 343-346 (2005)

[3] L. F. Y, L. J. M, "Chaotic lidar", IEEE J. Sel. Top. Quantum Electron. 10, 991-997 (2004)

[4] Y. Takushima and Y. C. Chung, "Optical reflectometry based on correlation detection and its application to the in-service monitoring of WDM passive optical network," Opt. Express 15, 5318-5326 (2007).

[5] Y. C. Wang, B. J. Wang, and A. B. Wang, "Chaotic correlation optical time domain reflectometry utilizing laser diode", IEEE Photon. Technol. Lett. 20, 1636-1638 (2008)

[6] M. A. Richards, [Fundamentals of Radar Signal Processing], McGraw-Hill, (2005) 